\title{
Metallicity Gradients in the Halos of Elliptical Galaxies
}

\section{Jenny E. Greene ${ }^{1}$, Chung-Pei $\mathrm{Ma}^{2}$, Andrew Goulding ${ }^{1}$, Nicholas J. McConnell ${ }^{3}$, John P. Blakeslee ${ }^{3}$, Timothy Davis ${ }^{4}$, Jens Thomas ${ }^{5}$}

\author{
${ }^{1}$ Department of Astrophysics, Princeton University, Princeton, NJ 08544, USA \\ ${ }^{2}$ Department of Astronomy, University of California, Berkeley, CA 94720, USA \\ ${ }^{3}$ Dominion Astrophysical Observatory, NRC Herzberg Institute of Astrophysics, Victoria, BC \\ V9E 2E7, Canada \\ ${ }^{4}$ Centre for Astrophysics Research, University of Hertfordshire, Hatfield, Herts AL10 9AB, UK \\ ${ }^{5}$ Max Planck-Institute for Extraterrestrial Physics, Giessenbachstr. 1, D-85741 Garching, \\ Germany
}

\begin{abstract}
We discuss the stellar halos of massive elliptical galaxies, as revealed by our ambitious integral-field spectroscopic survey MASSIVE. We show that metallicity drops smoothly as a function of radius out to $\sim 2.5 R_{e}$, while the $[\alpha / \mathrm{Fe}]$ abundance ratios stay flat. The stars in the outskirts likely formed rapidly (to explain the high ratio of alpha to $\mathrm{Fe}$ ) but in a relatively shallow potential (to explain the low metallicities). This is consistent with expectations for a two-phase growth of massive galaxies, in which the second phase involves accretion of small satellites. We also show some preliminary study of the gas content of these most MASSIVE galaxies.
\end{abstract}

Keywords. galaxies: elliptical and lenticular, $\mathrm{cD}$, galaxies: evolution, galaxies: kinematics and dynamics, galaxies: stellar content

\section{Introduction}

Although they comprise the most massive galaxies in the present-day universe, the assembly history of elliptical galaxies remains poorly understood. In this contribution, we discuss our ongoing ambitious integral-field spectroscopic (IFS) survey, MASSIVE (Ma et al. 2014). Our goal is to observe the $\sim 100$ most massive early-type galaxies within $\sim 100 \mathrm{Mpc}$ with the large-format IFS instrument, the George and Cynthia Mitchell Visible Integral Replicable Unit Prototype (hereafter the Mitchell spectrograph Hill et al. 2008).

Our survey is designed to complement existing IFS studies focused on early-type galaxies, and in particular ATLAS ${ }^{3 \mathrm{D}}$ (Cappellari et al. 2011). We complement their work in two important ways. First, the Mitchell spectrograph has a field of view of 2 arcmin, which allows us to investigate the stellar kinematics and stellar populations of our galaxies to beyond twice their effective radius. With this wide radial coverage, we are able to probe the dark matter halo potential with our stellar kinematics, as well as measure radial gradients in stellar populations out to large radius. Second, we survey galaxies to a volume limit of $107 \mathrm{Mpc}$ (to include the Coma cluster) and thus probe the very massive end of the galaxy mass function. We show the difference in sample demographics between the two surveys in Figure 1. We show a representative sample of galaxy images from MASSIVE in Figure 2. 


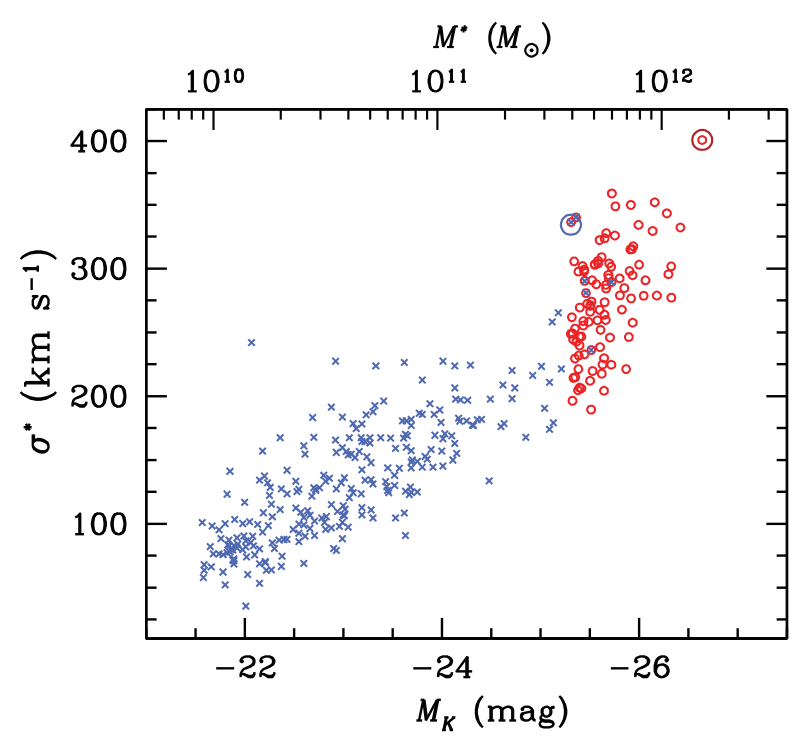

Figure 1. Adapted from Ma et al. 2014. We plot the stellar velocity dispersion versus the absolute magnitude (Faber \& Jackson 1976). In blue crosses, we show the ATLAD ${ }^{3 \mathrm{D}}$ galaxies while the MASSIVE galaxies are shown as red open circles. For context, we highlight in large circles NGC 4889 (red) and M87 (blue).
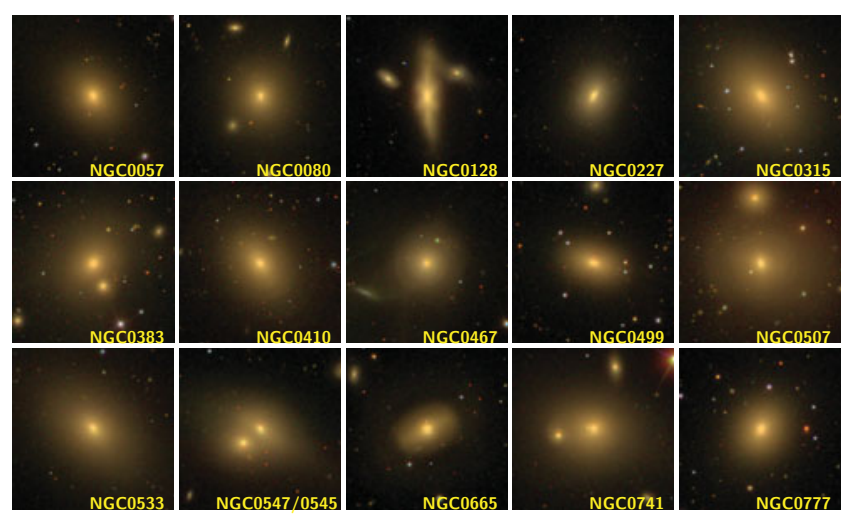

Figure 2. Three-color images from the SDSS, adapted from Ma et al. 2014.

\section{The Sample}

The MASSIVE sample contains 116 early-type galaxies in the northern sky with distance $D<108 \mathrm{Mpc}$ and absolute $K$-band magnitude $M_{K}<-25.3 \mathrm{mag}$ (stellar mass $\left.M^{*}>10^{11.5} M_{\odot}\right)$. The survey volume includes the Coma Cluster and is more than an order of magnitude larger than that probed by ATLAS ${ }^{3 \mathrm{D}}$, enabling us to obtain a statistical sample of early-type galaxies at the highest end of the galaxy mass function. The survey galaxies are selected based on their total stellar mass via the $K$-band luminosities from 2MASS. Details of the distance determination, morphological cut, and other selection criteria are described in Ma et al. (2014). 

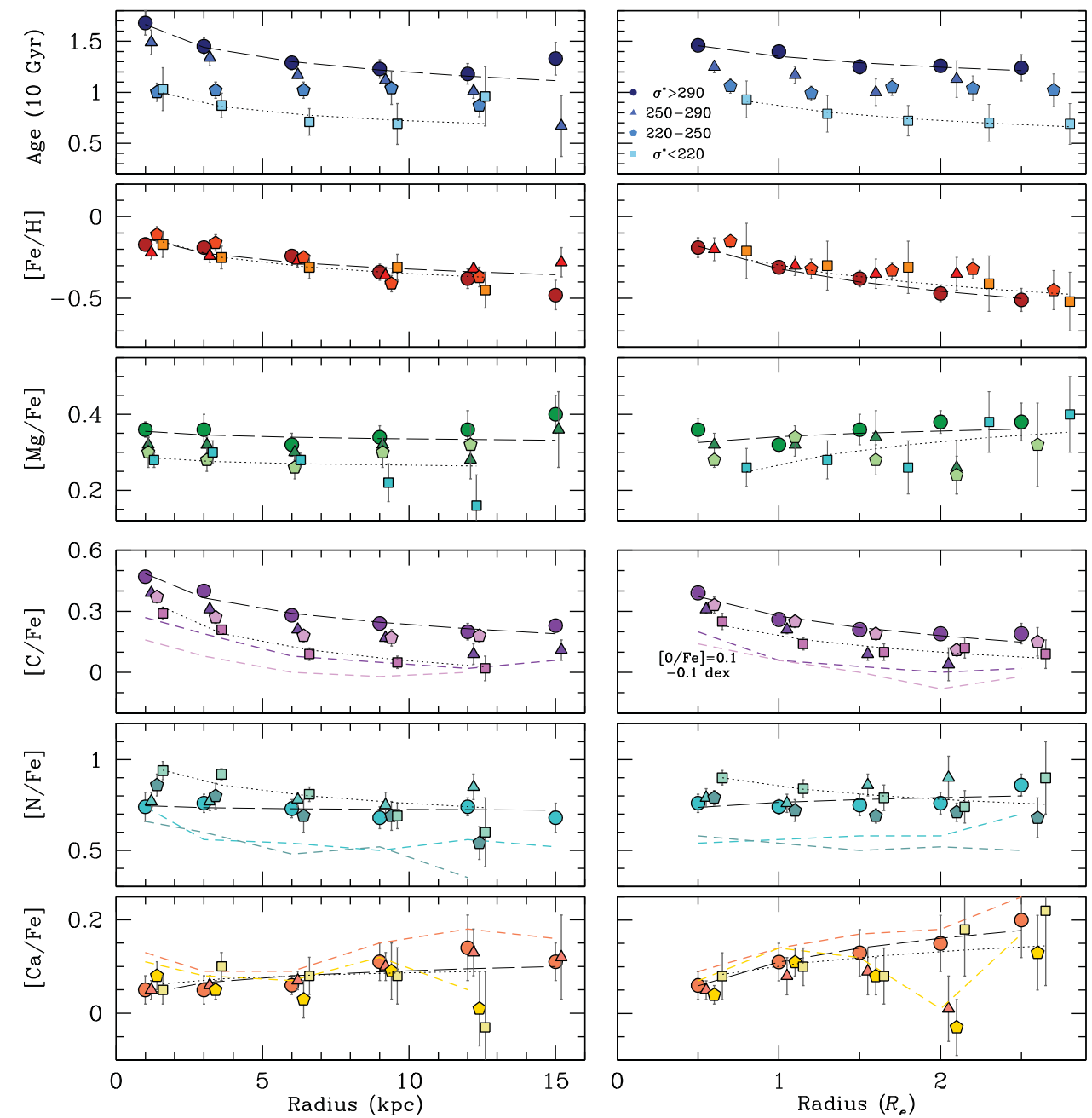

Figure 3. Radial gradients in age, $[\mathrm{Fe} / \mathrm{H}],[\mathrm{Mg} / \mathrm{Fe}],[\mathrm{C} / \mathrm{Fe}],[\mathrm{N} / \mathrm{Fe}]$, and $[\mathrm{Ca} / \mathrm{Fe}]$, adapted from Greene et al. (2015). The measurements are made on four sets of stacked spectra binned on stellar velocity dispersion (see figure key), and are shown as a function of $R$ in kpc (left) or $R / R_{e}$. The lines are power-law fits (see Greene et al. 2015). Note the decline with radius in $[\mathrm{Fe} / \mathrm{H}]$ and $[\mathrm{C} / \mathrm{Fe}]$ in contrast with the radially constant age, $[\mathrm{Mg} / \mathrm{Fe}],[\mathrm{N} / \mathrm{Fe}]$, and $[\mathrm{Ca} / \mathrm{Fe}]$. To indicate systematic errors in the light elements due to the unknown oxygen abundance, we also show the resulting models assuming $[\mathrm{O} / \mathrm{Fe}]=0.1$ rather than the default $[\mathrm{O} / \mathrm{Fe}]=0.5$ (keeping $[\mathrm{O} / \mathrm{Fe}]$ constant with radius in both cases $)\left(\sigma_{*}>290 \mathrm{~km} \mathrm{~s}^{-1}\right.$ coadd in dash and $220<\sigma_{*}<290$ $\mathrm{km} \mathrm{s}^{-1}$ in dot-dash). The $[\mathrm{C} / \mathrm{Fe}]$ lines with alternate oxygen abundance have been offset by -0.1 dex for presentation purposes.

\section{Stellar Population Gradients}

Radial gradients in stellar populations can elucidate when, how rapidly, and in what environment, the stars in galaxy outskirts were formed (e.g., White 1980; Kobayashi 2004; Greene et al. 2013; Hirschmann et al. 2015). At the same time, the kinematics of the stars (e.g., $V / \sigma_{*}$, the level of radial anisotropy, etc.) may provide insight into how these stars joined the galaxy (e.g., Wu et al. 2014; Arnold et al. 2014; Raskutti et al. 2014; Naab et al. 2014; Röttgers et al. 2014). We focus most on the former measurements here. 


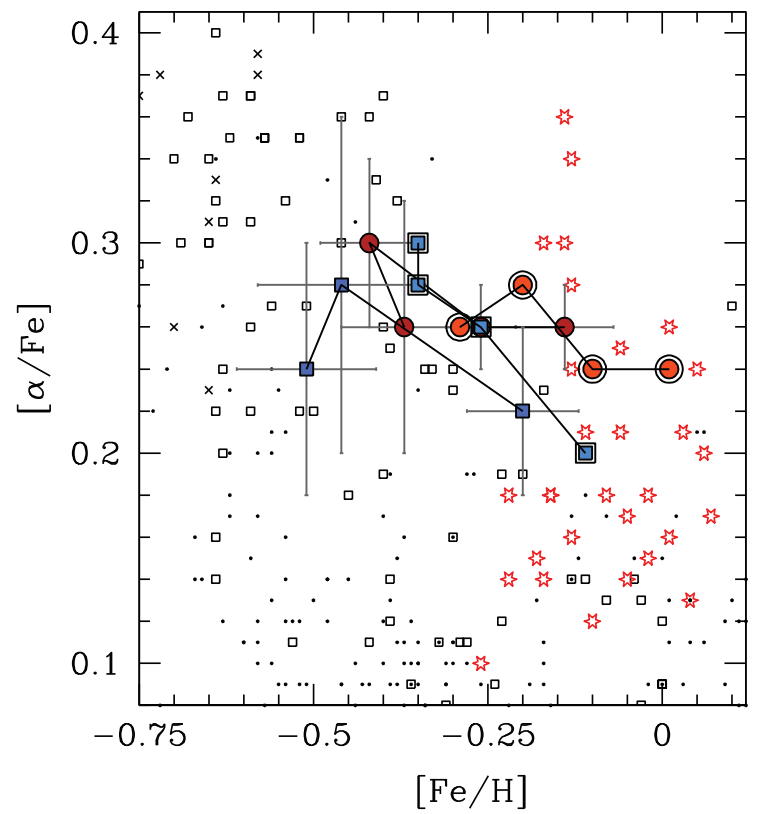

Figure 4. Adapted from Greene et al. (2013), we show galaxy centers from Graves et al. (2009) in red stars, well-aligned with the centers of our galaxies (large red circles and blue squares). As we follow the massive galaxy tracks outward from the locus of red stars, the radius grows. However, the $[\alpha / \mathrm{Fe}]$ stays roughly constant (to marginally rising) while the $[\mathrm{Fe} / \mathrm{H}]$ drops outward. For reference, we show Milky Way stars from Venn et al. (2004), including disk stars (small dots), thick disk stars (open squares), and halo stars (crosses).

Historically, measuring reliable stellar population parameters beyond the half-light radius of galaxies has been quite a challenge. Resolved stellar population studies, beautifully discussed at this meeting, are only able to reach a few of the nearest early-type galaxies (e.g., Kalirai et al. 2006; Harris et al. 1999; Rejkuba et al. 2005; Harris et al. 2007; Crnojević et al. 2013; Pastorello et al. 2014; Peacock et al. 2015; Williams et al. 2015), while only a handful of existing observations probe such a wide radial range (Carollo et al. 1993; Carollo \& Danziger 1994; Mehlert et al. 2003; Kelson et al. 2006; Spolaor et al. 2010; Pu et al. 2010; Pu \& Han 2011; Weijmans et al. 2009; Murphy et al. 2011). Imaging surveys have also made great strides recently using stacking techniques (e.g., D'Souza et al. 2014), which complement our approach.

The IFS data allows us to radially average our data in elliptical annuli and boost the signal-to-noise relative to classic long-slit techniques. In Figure 3 we show the average radial gradients in galaxies binned on different properties (here we show bins of stellar velocity dispersion Greene et al. 2015). We find no significant gradient in age, gently declining $[\mathrm{Fe} / \mathrm{H}]$, and flat radial behavior in $[\alpha / \mathrm{Fe}]$.

It is useful to look at the radial behavior of the galaxies in the oft-used $[\alpha / \mathrm{Fe}]$ vs $[\mathrm{Fe} / \mathrm{H}]$ plane (Figure 4). Here we see that the stars in the outskirts of massive elliptical galaxies share most in common with thin-disk stars in our own galaxy. This makes sense if the stars were formed rapidly (at high redshift) in a relatively shallow potential well.

\subsection{Dependence on Group Richness}

In Greene et al. (2015) we leverage the large MASSIVE sample to investigate the possible dependence of radial gradients on the richness of the large-scale galaxy environment. Specifically, despite being a massive galaxy survey, roughly $40 \%$ of the galaxies in MAS- 


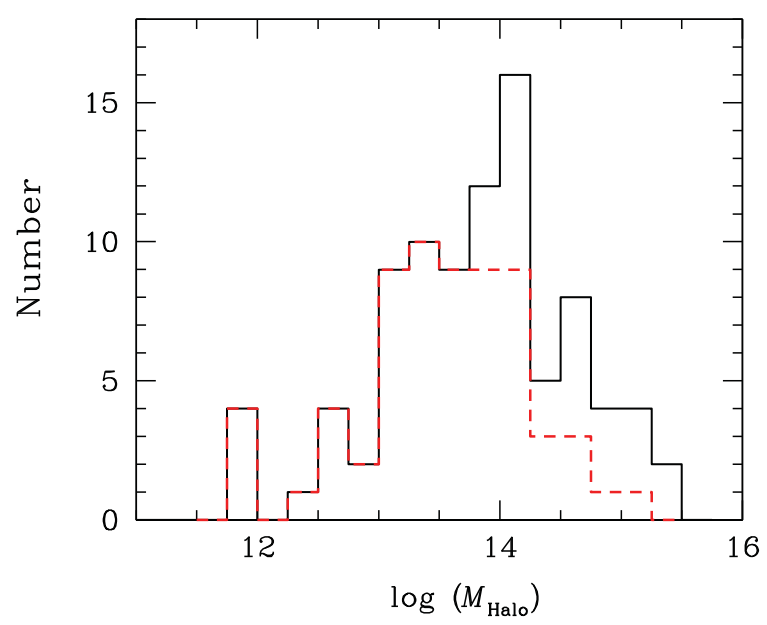

Figure 5. Distribution of dark matter halo masses for the 90 MASSIVE galaxies that reside in groups in the HDC catalog (black histogram), from Crook et al. Among the 90, 65 are the brightest group galaxies (BGGs) in their respective groups (red histogram).

SIVE live in isolated environments (Figure 5). Using the group catalog of Crook et al. (2007), we divide the galaxies into groups (with more than three members) and field galaxies. By forcing identical distributions in $\sigma_{*}$, we are able to investigate whether, at fixed $\sigma_{*}$, there are differences in radial gradients in the cluster and the field (Figure 6).

We see only slight differences in the radial gradients as a function of group richness. At higher density (and fixed $\sigma_{*}$ ) the galaxies are slightly older, slightly $[\mathrm{Fe} / \mathrm{H}]$ poorer, and slightly alpha-enhanced. We might expect such trends if galaxies in richer environments form earlier, but cannot rule out the alternate possibility that at fixed $\sigma_{*}$, galaxies in richer environments may be smaller (Greene et al. 2015). Trying to focus only on the brightest galaxies in a group cut down our sample size considerably.

\section{Gas}

We have also been working to characterize the gas content of the MASSIVE galaxies. The mass and phase of the gas provides additional insight into the growth mechanisms of these galaxies, as well as providing complementary dynamical tracers.

The dominant phase of gas in massive elliptical galaxies is hot, X-ray emitting gas. Study of the distribution, temperature, and luminosity of the hot gas (A. Goulding et al. in preparation; Figure 7) both allows us to understand the origin and kinematics of the gas, and also study the dark matter halo mass. Disturbances in the X-ray emission are strong indicators of recent merger or nuclear activity. Combining our measurements with those from ATLAS $^{3 \mathrm{D}}$ (e.g., Sarzi et al. 2013; Kim \& Fabbiano 2015) will give us a very broad baseline in stellar mass, $\sigma_{*}$, rotation, and halo mass, with which to study the origin and kinematics of the hot gas (e.g., Negri et al. 2014).

Although elliptical galaxies are gas poor, they are not always gas free (e.g., Knapp et al. 1989). We have initiated a CO survey, and in our initial pilot survey detected six of the eleven galaxies that we targeted (Davis et al. submitted). We have a more comprehensive search ongoing with the IRAM-30m telescope (PI Davis) to map out the CO detection fraction as a function of mass and $\sigma_{*}$. From our pilot survey, we find intriguing evidence that the most massive galaxies rotate significantly faster than expected from the TullyFisher relation of less massive early-type galaxies (Davis et al. submitted). 

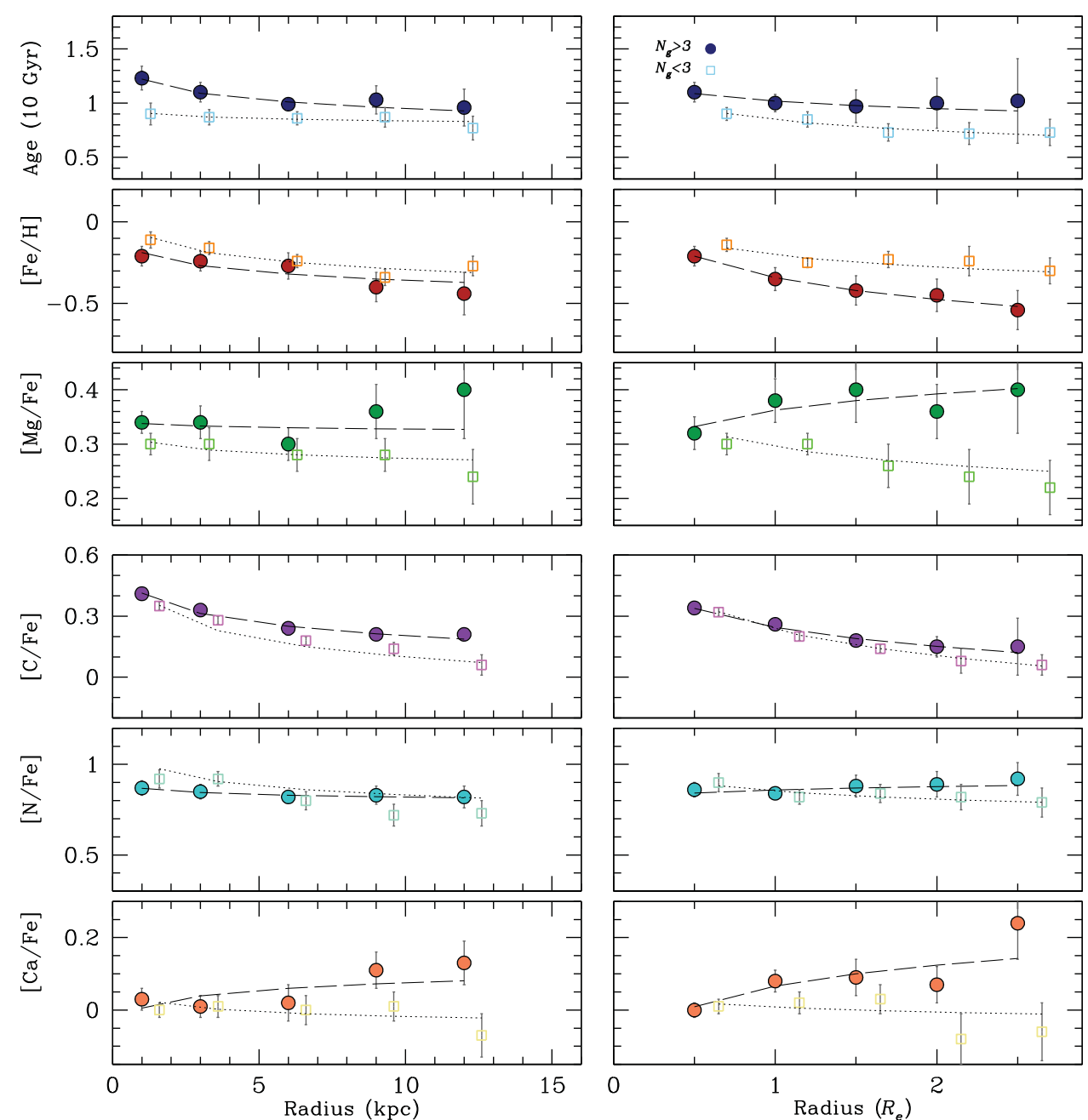

Figure 6. Radial gradients in age, $[\mathrm{Fe} / \mathrm{H}],[\mathrm{Mg} / \mathrm{Fe}],[\mathrm{C} / \mathrm{Fe}],[\mathrm{N} / \mathrm{Fe}]$, and $[\mathrm{Ca} / \mathrm{Fe}]$ as above, but now in bins of group richness, controlling for the distribution of $\sigma_{*}$. Low density (open squares) have three or fewer neighbors with $L>L^{*}$, while filled circles include everything else. The lines represent power-law fits to the gradients.

To complement the study of the molecular gas, we are also mapping the ionized gas using [O II] emission in our Mitchell cubes (Pandya et al. in prep). Thus far, we detect roughly one-fifth of the galaxies, with a range of morphologies ranging from organized rotating disks to patchy, disorganized regions with no ordered rotation. In a few cases, the gas extends to beyond four times the effective radius, potentially signaling cooling flows in these systems. We find that the detection fraction is a strong function of $\sigma_{*}$. Note that nearly all ATLAS ${ }^{3 \mathrm{D}}$ galaxies are detected in ionized gas (Sarzi et al. 2010) and that the angular momentum of the ionized and CO gas tend to align when they are both detected (Davis et al. 2011).

\section{Future Work}

To go further in our understanding of the assembly of elliptical galaxies, we must combine our stellar population measurements with the kinematic information that we can also extract from our spectra (M. Veale et al. in prep). We will ask whether there 


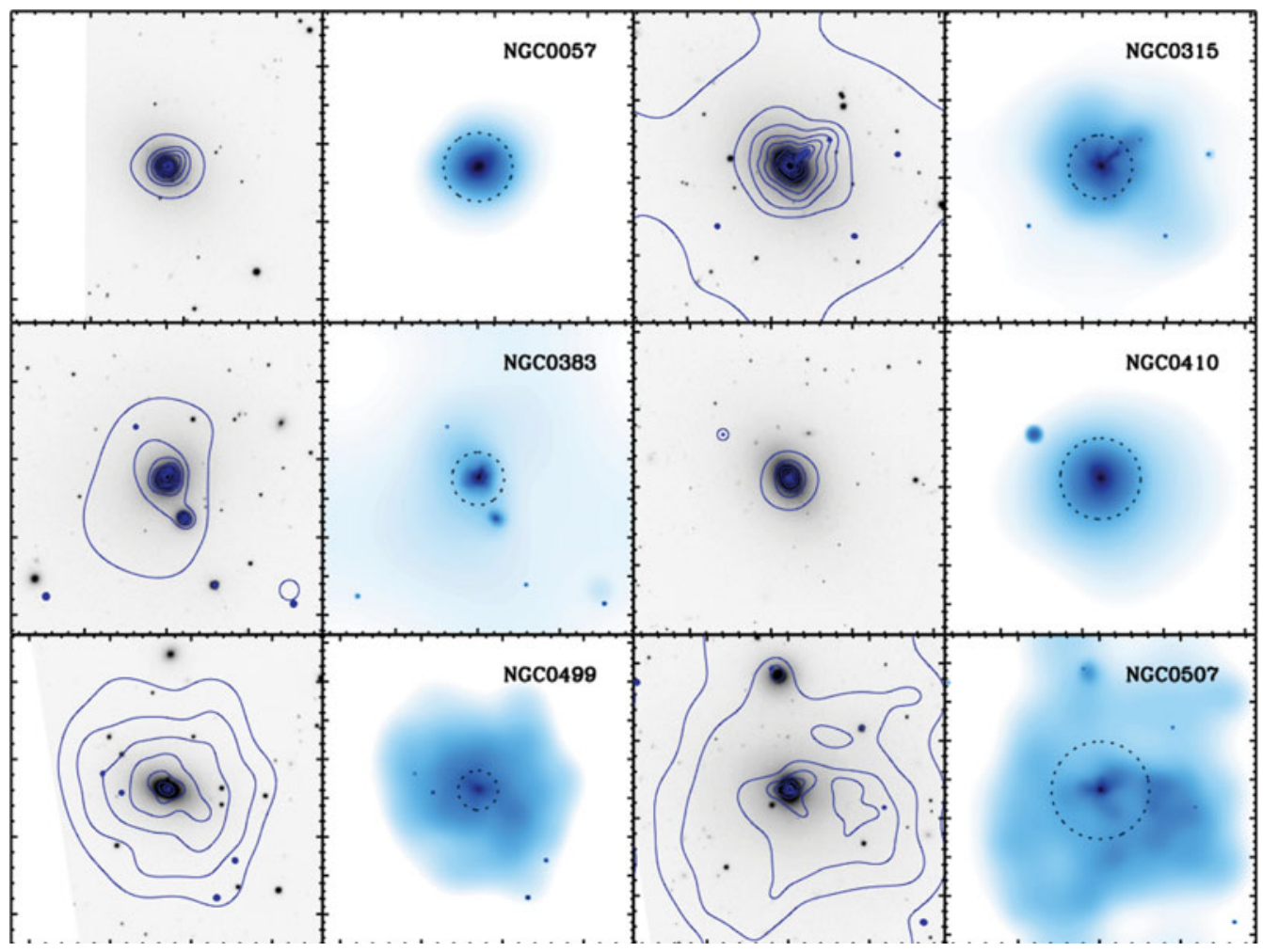

Figure $7.4 \times 4$ arcminute cutouts of the MASSIVE galaxies with publicly available archival Chandra X-ray observations performed with the ACIS instrument, adapted from Goulding et al. in preparation. Left column shows the optical $i$-band SDSS DR12 Atlas image, logarithmic X-ray contours are overlaid in solid blue. The logarithm of the adaptively-smoothed and vignetting and exposure-corrected Chandra ACIS-S images are shown to the right of the optical images. Dashed circles represent one effective radius derived from optical imaging (see Ma et al. 2014 for details).

is any relationship between the angular momentum content of the stars at large radius and the stellar population gradients. We will use the molecular and ionized gas as an independent probe of the enclosed mass and investigate the initial mass function of these galaxies. Finally, we will use our upcoming HST observations (PI Blakeslee) to look for links between galaxy structure and stellar populations.

\section{References}

Arnold, J. A., Romanowsky, A. J., Brodie, J. P., Forbes, D. A., Strader, J., Spitler, L. R., Foster, C., Blom, C., Kartha, S. S., Pastorello, N., Pota, V., Usher, C., \& Woodley, K. A. 2014, ApJ, 791, 80

Cappellari, M., Emsellem, E., Krajnović, D., McDermid, R. M., Serra, P., Alatalo, K., Blitz, L., Bois, M., Bournaud, F., Bureau, M., Davies, R. L., Davis, T. A., de Zeeuw, P. T., Khochfar, S., Kuntschner, H., Lablanche, P.-Y., Morganti, R., Naab, T., Oosterloo, T., Sarzi, M., Scott, N., Weijmans, A.-M., \& Young, L. M. 2011, MNRAS, 416, 1680

Carollo, C. M., \& Danziger, I. J. 1994, MNRAS, 270, 743

Carollo, C. M., Danziger, I. J., \& Buson, L. 1993, MNRAS, 265, 553

Crnojević, D., Ferguson, A. M. N., Irwin, M. J., Bernard, E. J., Arimoto, N., Jablonka, P., \& Kobayashi, C. 2013, MNRAS, 432, 832

Crook, A. C., Huchra, J. P., Martimbeau, N., Masters, K. L., Jarrett, T., \& Macri, L. M. 2007, ApJ, 655, 790 
Davis, T. A., et al. 2011, MNRAS, 417, 882

D'Souza, R., Kauffman, G., Wang, J., \& Vegetti, S. 2014, MNRAS, 443, 1433

Faber, S. M., \& Jackson, R. E. 1976, ApJ, 204, 668

Graves, G. J., Faber, S. M., \& Schiavon, R. P. 2009, ApJ, 693, 486

Greene, J. E., Janish, R., Ma, C.-P., McConnell, N. J., Blakeslee, J. P., Thomas, J., \& Murphy, J. D. 2015, ApJ, 807, 11

Greene, J. E., Murphy, J. D., Graves, G. J., Gunn, J. E., Raskutti, S., Comerford, J. M., \& Gebhardt, K. 2013, ApJ, 776, 64

Harris, G. L. H., Harris, W. E., \& Poole, G. B. 1999, AJ, 117, 855

Harris, W. E., Harris, G. L. H., Layden, A. C., \& Wehner, E. M. H. 2007, ApJ, 666, 903

Hill, G. J., et al. 2008, in Society of Photo-Optical Instrumentation Engineers (SPIE) Conference Series, Vol. 7014, Society of Photo-Optical Instrumentation Engineers (SPIE) Conference Series

Hirschmann, M., Naab, T., Ostriker, J. P., Forbes, D. A., Duc, P.-A., Davé, R., Oser, L., \& Karabal, E. 2015, MNRAS, 449, 528

Kalirai, J. S., et al. 2006, ApJ, 648, 389

Kelson, D. D., Illingworth, G. D., Franx, M., \& van Dokkum, P. G. 2006, ApJ, 653, 159

Kim, D.-W., \& Fabbiano, G. 2015, ApJ, accepted (arXiv:1504.00899)

Knapp, G. R., Guhathakurta, P., Kim, D.-W., \& Jura, M. A. 1989, ApJS, 70, 329

Kobayashi, C. 2004, MNRAS, 347, 740

Ma, C.-P., Greene, J. E., McConnell, N., Janish, R., Blakeslee, J. P., Thomas, J., \& Murphy, J. D. 2014, ApJ, 795, 158

Mehlert, D., Thomas, D., Saglia, R. P., Bender, R., \& Wegner, G. 2003, A\&6A, 407, 423

Murphy, J. D., Gebhardt, K., \& Adams, J. J. 2011, ApJ, 729, 129

Naab, T., Oser, L., Emsellem, E., Cappellari, M., Krajnović, D., McDermid, R. M., Alatalo, K., Bayet, E., Blitz, L., Bois, M., Bournaud, F., Bureau, M., Crocker, A., Davies, R. L., Davis, T. A., de Zeeuw, P. T., Duc, P.-A., Hirschmann, M., Johansson, P. H., Khochfar, S., Kuntschner, H., Morganti, R., Oosterloo, T., Sarzi, M., Scott, N., Serra, P., Ven, G. v. d., Weijmans, A., \& Young, L. M. 2014, MNRAS, 444, 3357

Negri, A., Ciotti, L., \& Pellegrini, S. 2014, MNRAS, 439, 823

Pastorello, N., Forbes, D. A., Foster, C., Brodie, J. P., Usher, C., Romanowsky, A. J., Strader, J., \& Arnold, J. A. 2014, MNRAS, 442, 1003

Peacock, M. B., Strader, J., Romanowsky, A. J., \& Brodie, J. P. 2015, ApJ, 800, 13

Pu, S.-B., \& Han, Z.-W. 2011, Research in Astronomy and Astrophysics, 11, 909

Pu, S. B., Saglia, R. P., Fabricius, M. H., Thomas, J., Bender, R., \& Han, Z. 2010, A\&AA, 516, A4

Raskutti, S., Greene, J. E., \& Murphy, J. D. 2014, ApJ, 786, 23

Rejkuba, M., Greggio, L., Harris, W. E., Harris, G. L. H., \& Peng, E. W. 2005, ApJ, 631, 262

Röttgers, B., Naab, T., \& Oser, L. 2014, MNRAS, 445, 1065

Sarzi, M., Alatalo, K., Blitz, L., Bois, M., Bournaud, F., Bureau, M., Cappellari, M., Crocker, A., Davies, R. L., Davis, T. A., de Zeeuw, P. T., Duc, P.-A., Emsellem, E., Khochfar, S., Krajnović, D., Kuntschner, H., Lablanche, P.-Y., McDermid, R. M., Morganti, R., Naab, T., Oosterloo, T., Scott, N., Serra, P., Young, L. M., \& Weijmans, A.-M. 2013, MNRAS, 432,1845

Sarzi, M., et al. 2010, MNRAS, 402, 2187

Spolaor, M., Kobayashi, C., Forbes, D. A., Couch, W. J., \& Hau, G. K. T. 2010, MNRAS, 408, 272

Venn, K. A., Irwin, M., Shetrone, M. D., Tout, C. A., Hill, V., \& Tolstoy, E. 2004, AJ, 128, 1177

Weijmans, A.-M., et al. 2009, MNRAS, 398, 561

White, S. D. M. 1980, MNRAS, 191, 1P

Williams, B. F., Dalcanton, J. J., Gilbert, E. F. B. M., Guhathakurta, P., Dorman, C., Lauer, T. R., Seth, A. C., Kalirai, J. S., Rosenfield, P., \& Girardi, L. 2015, ApJ, accepted (arXiv:1501.06631)

Wu, X., Gerhard, O., Naab, T., Oser, L., Martinez-Valpuesta, I., Hilz, M., Churazov, E., \& Lyskova, N. 2014, MNRAS, 438, 2701 